\title{
A Link Between Riemann Invariants and Frequency Domain Approaches for Boundary Control of Open Channel Flow
}

\author{
Xavier Litrico and Vincent Fromion
}

\begin{abstract}
Open channel flow is traditionally described by hyperbolic conservation laws (the Saint-Venant equations), that can be controlled using boundary conditions. For horizontal frictionless channels, a classical approach consists in using the characteristic form to diagonalize the equations, using socalled Riemann invariants. This elegant approach is much more difficult to apply when friction and slope are not zero, i.e. in the vast majority of cases. On the other hand, a Laplace based method enables to diagonalize the system with nonzero slope and friction, but in the frequency domain. This paper enlightens a link between both methods, showing that the frequency domain method can be considered as an extension of the Riemman invariants form for channels with non zero slope and friction. As an application, we derive explicit expressions for the boundary controls solving the motion planning problem.
\end{abstract}

\section{INTRODUCTION}

\section{A. Motivation}

This work is motivated by the problem of controlling an open channel represented by Saint-Venant equations. These hyperbolic partial differential equations describe the dynamics of open channel hydraulic systems, e.g. rivers, irrigation or drainage canals, sewers, etc., assuming one dimensional flow.

The distributed feature of these systems is usually taken into account by using a Riemann invariants approach [2], which uses an adequate change of variables to diagonalize the system. However, this solution is only valid for a specific case, corresponding to rectangular horizontal frictionless channels around a uniform flow regime. This main limitation of the Riemann invariants method has lead to consider an alternative method based on frequency domain approach [5], [4], [6]. Such a method is very close to the one classically used by control engineers: the nonlinear PDE is first linearized around a stationary regime, then the Laplace transform is used to consider the linearized PDE in the frequency domain, and classical frequency domain tools are used to design controllers, in a very similar way as when the system is represented by finite dimensional transfer functions.

An interesting feature of this approach is that it naturally takes into account the effect of slope and friction on the hyperbolic equation, while this is not easily done with Riemann invariants [8]. Indeed, the characteristic form leads to Riemann invariants only when the channel is horizontal

$\mathrm{X}$. Litrico is with UMR G-EAU, Cemagref, Montpellier, France. xavier.litrico@cemagref.fr.

V. Fromion is with INRA-MIG, Jouy-en-Josas, France. vincent.fromionejouy.inra.fr. and there is no friction. In that case, the characteristic form leads to diagonalize the system.

On the other hand, the frequency domain approach also enables to diagonalize the system with nonzero slope and friction, but in the frequency domain.

We investigate in this article the link between both approaches and provide closed-form expressions for a dynamic change of variable that extends the Riemann invariants approach to the case where the open channel has nonzero slope and friction.

As an application, we derive expressions for the boundary controls solving the motion planning problem, which has been considered in other applications [7]. We also extend the stability condition for feedback control obtained using Riemann invariants methods to the case of channels with slope and friction.

\section{B. Control Problem}

The linearized Saint-Venant equations express mass and momentum conservation in an open channel around a steady flow defined by a discharge $Q_{0}$ and a water depth $Y_{0}$.

The linearized equations are given by:

$$
\frac{\partial \xi}{\partial t}+\mathbf{A} \frac{\partial \xi}{\partial x}+\mathbf{B} \xi=0
$$

where $\xi(x, t)=(a(x, t), q(x, t))^{T}:[0, L] \times[0,+\infty) \rightarrow$ $\Omega \in \mathbb{R}^{2}$ is the state of the system and $\mathbf{A}=\left(\begin{array}{cc}0 & 1 \\ \alpha \beta & \alpha-\beta\end{array}\right)$, $\mathbf{B}=\left(\begin{array}{cc}0 & 0 \\ -\gamma & \delta\end{array}\right)$

The parameters are given by: $\alpha=C_{0}+V_{0}, \beta=C_{0}-V_{0}$, $\gamma=g(1+\kappa) S_{b}, \delta=\frac{2 g S_{b}}{V_{0}}, \kappa=\frac{7}{3}-\frac{4 A_{0}}{3 T_{0} P_{0}} \frac{\partial P_{0}}{\partial Y}$, where $V_{0}$ is the mean velocity, $C_{0}$ the celerity, $F_{0}=V_{0} / C_{0}$ is the Froude number $\left(F_{0}<1\right), S_{b}$ the bottom slope, $\kappa$ a coefficient linked to the geometry of the channel, $T_{0}$ the top width.

We consider the solutions of the Cauchy problem for the system (1) over $[0,+\infty) \times[0, L]$ under an initial condition $\xi(0, x)=\xi_{0}(x), x \in[0, L]$ and two boundary conditions of the form $q(t, 0)=q_{0}(t)$ and $q(t, L)=q_{L}(t), t \in[0,+\infty)$.

\section{Horizontal Frictionless CASE}

We deal in this section with the special case where $\delta=$ $\gamma=0$, corresponding to horizontal frictionless open channel flow.

\section{A. Characteristic Form}

Let us introduce the following change of variable, which corresponds to the characteristic form of the linearized 
equations:

$$
\begin{aligned}
& \chi_{1}(x, t)=q(x, t)+\beta T_{0} y(x, t) \\
& \chi_{2}(x, t)=q(x, t)-\alpha T_{0} y(x, t)
\end{aligned}
$$

Using a matrix notation, and the fact that $\xi=\left(T_{0} y, q\right)^{T}$, we therefore have:

$$
\chi(x, t)=\mathbf{X} \xi(x, t)
$$

with $\mathbf{X}=\left(\begin{array}{cc}\beta & 1 \\ -\alpha & 1\end{array}\right)$

This change of variable enables us to diagonalize matrix A as follows:

$$
\mathbf{A}=\mathbf{X}^{-1} \mathbf{D X}
$$

with

$$
\mathbf{D}=\left(\begin{array}{cc}
\alpha & 0 \\
0 & -\beta
\end{array}\right)
$$

The equation (1) can then be rewritten as:

$$
\frac{\partial \chi}{\partial t}+\mathbf{D} \frac{\partial \chi}{\partial x}=0
$$

This change of variable corresponds to the characteristic form of the equations. Indeed, the new variable $\chi(x, t)$ verifies:

$$
\begin{aligned}
& \frac{d \chi_{1}}{d t}\left(x_{1}(t), t\right)=0 \text { along } \frac{d x_{1}}{d t}(t)=\alpha \\
& \frac{d \chi_{2}}{d t}\left(x_{2}(t), t\right)=0 \text { along } \frac{d x_{2}}{d t}(t)=-\beta
\end{aligned}
$$

The variables $\chi_{1}(x, t)$ and $\chi_{2}(x, t)$ are constant along the characteristic lines defined by Eqs. (4a) and (4b). They are called Riemann invariants of the system (1).

Let us now use the Laplace transform to compute the transfer matrix of the system.

\section{B. Frequency Domain Approach}

We denote in the following $\mathcal{L}(f)=\hat{f}(s)$ the Laplace transform of a function $f(t)$. To derive the transfer matrix, we apply Laplace transform on Eq. (3), which leads to:

$$
\frac{\partial \hat{\chi}(x, s)}{\partial x}=-s \mathbf{D}^{-1} \hat{\chi}(x, s)+\mathbf{D}^{-1} \chi_{0}(x)
$$

where $\chi_{0}(x)=\mathbf{X} \xi_{0}(x)$ is the initial condition expressed in the characteristic variables.

The general solution of this ordinary differential equation in $x$ is then given by:

$\hat{\chi}(x, s)=\Phi(x, s) \hat{\chi}(0, s)+\Phi(x, s) \int_{0}^{x} \Phi(v, s)^{-1} \mathbf{D}^{-1} \chi_{0}(v) d v$

with $\Phi(x, s)=e^{-s \mathbf{D}^{-1} x}=\left(\begin{array}{cc}e^{-\frac{s x}{\alpha}} & 0 \\ 0 & e^{\frac{s x}{\beta}}\end{array}\right)$.

The components of the solution can then be expressed as:

$$
\begin{aligned}
& \hat{\chi}_{1}(x, s)=e^{-s \frac{x}{\alpha}} \hat{\chi}_{1}(0, s)+\bar{\chi}_{10}(x, s) \\
& \hat{\chi}_{2}(x, s)=e^{-s \frac{L-x}{\beta}} \hat{\chi}_{2}(L, s)+\bar{\chi}_{20}(x, s)
\end{aligned}
$$

with

$$
\begin{aligned}
& \bar{\chi}_{10}(x, s)=\frac{1}{\alpha} \int_{0}^{x} e^{-s \frac{x-v}{\alpha}} \chi_{10}(v) d v \\
& \bar{\chi}_{20}(x, s)=\frac{1}{\beta} \int_{x}^{L} e^{s \frac{x-v}{\beta}} \chi_{20}(v) d v
\end{aligned}
$$

Let us now define $\tau_{1}$ and $\tau_{2}$, the delays for downstream and upstream propagation, respectively:

$$
\begin{gathered}
\tau_{1}=\frac{L}{\alpha}=\frac{L}{C_{0}+V_{0}} \\
\tau_{2}=\frac{L}{\beta}=\frac{L}{C_{0}-V_{0}}
\end{gathered}
$$

Using Eqs. (7), we can now express the input-output transfer matrix in terms of the characteristics variables at the boundaries:

$$
\left(\begin{array}{c}
\hat{\chi}_{1}(L, s) \\
\hat{\chi}_{2}(0, s)
\end{array}\right)=G_{P}(s)\left(\begin{array}{c}
\hat{\chi}_{1}(0, s) \\
\hat{\chi}_{2}(L, s)
\end{array}\right)+\left(\begin{array}{c}
\bar{\chi}_{10}(L, s) \\
\bar{\chi}_{20}(0, s)
\end{array}\right)
$$

with $G_{P}(s)=\left(\begin{array}{cc}e^{-\tau_{1} s} & 0 \\ 0 & e^{-\tau_{2} s}\end{array}\right)$.

We see that in the horizontal frictionless case, the transfer matrix is diagonal in the characteristics variables, and only contains delays. This is consistent with the physical interpretation of the characteristics: the variable $\chi_{1}(x, t)$ can be expressed as a delayed function of its value at the upstream boundary $x=0$, and the variable $\chi_{2}(x, t)$ can be expressed as a delayed function of its value at the downstream boundary $x=L$. The flow is the result of the interaction of these two traveling waves.

1) Solution in the Time Domain: The solution (6) can be expressed in the time domain using the changes of variables $t_{1}=\frac{x-v}{\alpha}$ for the first integral of (8) and $t_{2}=\frac{v-x}{\beta}$ for the second one. This yields:

$$
\begin{aligned}
& \bar{\chi}_{10}(x, s)=\int_{0}^{\frac{x}{\alpha}} e^{-s t_{1}} \chi_{10}\left(x-\alpha t_{1}\right) d t_{1} \\
& \bar{\chi}_{20}(x, s)=\int_{0}^{\frac{L-x}{\beta}} e^{-s t_{2}} \chi_{20}\left(x+\beta t_{2}\right) d t_{2}
\end{aligned}
$$

We now observe that $\bar{\chi}_{10}(x, s)$ and $\bar{\chi}_{20}(x, s)$ are the Laplace transform of $\chi_{10}(x)$ and $\chi_{20}(x)$ on a truncated time interval, $\left[0, \frac{x}{\alpha}\right]$ for $\chi_{10}(x)$ and $\left[0, \frac{L-x}{\beta}\right]$ for $\chi_{20}(x)$. This is related to the fact that the initial condition has an influence in a given sector of the $(x, t)$ plane. Since we also know that the inverse Laplace transform of $e^{-\tau s} \hat{u}(s)$ is the delayed function $u(t-\tau)$, we obtain the following expressions in the time domain:

$$
\begin{aligned}
& \chi_{1}(x, t)= \begin{cases}\chi_{10}(x-\alpha t) & \text { if } x \geq \alpha t \\
\chi_{1}\left(0, t-\frac{x}{\alpha}\right) & \text { if } x<\alpha t\end{cases} \\
& \chi_{2}(x, t)= \begin{cases}\chi_{20}(x+\beta t) & \text { if } L-x \geq \beta t \\
\chi_{2}\left(L, t-\frac{L-x}{\beta}\right) & \text { if } L-x<\beta t\end{cases}
\end{aligned}
$$

We recover the classical solution obtained using the characteristics form. This result enables to show the link between the time domain and the frequency domain approaches. 


\section{NONZERO SLOPE AND FRICTION}

Nonzero slope and friction modify the characteristics equations: they induce a coupling which appears as a source term in the characteristics equations. But, as we will show below, it is still possible to eliminate this coupling by using a dynamic change of variable, which can easily be obtained in the Laplace domain.

Using the characteristics change of variable leads to:

$$
\frac{\partial \chi}{\partial t}+\mathbf{D} \frac{\partial \chi}{\partial x}+\mathbf{E} \chi=0
$$

where $\chi(x, t)=\mathbf{X} \xi(x, t)$ with $\xi(x, t)=$ $\left(T_{0} y(x, t), q(x, t)\right)^{T}, \mathbf{X}=\left(\begin{array}{cc}\beta & 1 \\ -\alpha & 1\end{array}\right), \mathbf{D}=\left(\begin{array}{cc}\alpha & 0 \\ 0 & -\beta\end{array}\right)$, and $\mathbf{E}=\mathbf{X B X}^{-1}$, with $\mathbf{B}=\left(\begin{array}{cc}0 & 0 \\ -\gamma & \delta\end{array}\right)$

In the horizontal frictionless case, the characteristics system was diagonal because matrix $\mathbf{B}$ was zero. In the uniform flow case with nonzero slope and friction, matrix $\mathbf{B}$ is non zero, and matrix $\mathbf{E}$ in Eq. (13) is non diagonal. Then, the solution in the time domain cannot be obtained as easily with the characteristics variables as in the horizontal frictionless case.

Still, the system can be diagonalized in the Laplace domain, as will be shown below. We now turn back to the original system (1).

\section{A. Derivation of the Transfer Matrix}

We apply Laplace transform to the linear partial differential equations (1), using the classical relation $\frac{\hat{d f}}{d t}=$ $s \hat{f}(s)-f(0)$, which yields:

$$
\frac{\partial \hat{\xi}(x, s)}{\partial x}=\mathcal{A}(s) \hat{\xi}(x, s)+\mathcal{B} \xi(x, 0)
$$

with

$$
\begin{aligned}
\mathcal{A}(s) & =\frac{1}{\alpha \beta}\left(\begin{array}{cc}
(\alpha-\beta) s+\gamma & -s-\delta \\
-\alpha \beta s & 0
\end{array}\right) \\
\mathcal{B} & =\frac{1}{\alpha \beta}\left(\begin{array}{cc}
(\beta-\alpha) & 1 \\
\alpha \beta & 0
\end{array}\right) .
\end{aligned}
$$

Let us diagonalize matrix $\mathcal{A}(s)$ :

$$
\mathcal{A}(s)=\mathcal{X}(s)^{-1} \mathcal{D}(s) \mathcal{X}(s)
$$

with $\mathcal{X}(s)=\left(\begin{array}{cc}\frac{s}{\lambda_{2}(s)} & 1 \\ \frac{s}{\lambda_{1}(s)} & 1\end{array}\right), \mathcal{D}(s)=\left(\begin{array}{cc}\lambda_{1}(s) & 0 \\ 0 & \lambda_{2}(s)\end{array}\right)$, $\mathcal{X}(s)^{-1}=\frac{\lambda_{1}(s) \lambda_{2}(s)}{s\left(\lambda_{1}(s)-\lambda_{2}(s)\right)}\left(\begin{array}{cc}1 & -1 \\ -\frac{s}{\lambda_{1}(s)} & \frac{s}{\lambda_{2}(s)}\end{array}\right)$, and where $\lambda_{1}(s)$ and $\lambda_{2}(s)$ are the eigenvalues of $\mathcal{A}(s)$, given by:

$$
\begin{aligned}
& \lambda_{1}(s)=\frac{(\alpha-\beta) s+\gamma-\sqrt{d(s)}}{2 \alpha \beta} \\
& \lambda_{2}(s)=\frac{(\alpha-\beta) s+\gamma+\sqrt{d(s)}}{2 \alpha \beta}
\end{aligned}
$$

with $d(s)=(\alpha+\beta)^{2} s^{2}+2[(\alpha-\beta) \gamma+2 \alpha \beta \delta] s+\gamma^{2}$.

In the following, we will factorize $d(s)$ as follows: $d(s)=$ $(\alpha+\beta)^{2}\left[(s+b)^{2}-a^{2}\right]$, with $a^{2}=\frac{4 \alpha \beta(\alpha \delta-\gamma)(\gamma+\beta \delta)}{(\alpha+\beta)^{4}}$ and $b=\frac{(\alpha-\beta) \gamma+2 \alpha \beta \delta}{(\alpha+\beta)^{2}}$.

For subcritical flow, it is easy to prove that $0<a<b$.

\section{B. Distributed Transfer Matrix}

Once matrix $\mathcal{A}(s)$ is diagonalized, the distributed transfer matrix relating the water depth $\hat{y}(x, s)$ and the discharge $\hat{q}(x, s)$ at any point $x$ in the canal pool to the upstream and downstream discharges is obtained as:

$$
\left(\begin{array}{l}
\hat{y}(x, s) \\
\hat{q}(x, s)
\end{array}\right)=G(x, s)\left(\begin{array}{c}
\hat{q}(0, s) \\
\hat{q}(L, s)
\end{array}\right)
$$

with

$$
\begin{aligned}
g_{11}(x, s) & =\frac{\lambda_{2}(s) e^{\lambda_{2}(s) x+\lambda_{1}(s) L}-\lambda_{1}(s) e^{\lambda_{1}(s) x+\lambda_{2}(s) L}}{T_{0} s\left(e^{\lambda_{2}(s) L}-e^{\lambda_{1}(s) L}\right)} \\
g_{12}(x, s) & =\frac{\lambda_{1}(s) e^{\lambda_{1}(s) x}-\lambda_{2}(s) e^{\lambda_{2}(s) x}}{T_{0} s\left(e^{\lambda_{2}(s) L}-e^{\lambda_{1}(s) L}\right)} \\
g_{21}(x, s) & =\frac{e^{\lambda_{1}(s) x+\lambda_{2}(s) L}-e^{\lambda_{2}(s) x+\lambda_{1}(s) L}}{e^{\lambda_{2}(s) L}-e^{\lambda_{1}(s) L}} \\
g_{22}(x, s) & =\frac{e^{\lambda_{2}(s) x}-e^{\lambda_{1}(s) x}}{e^{\lambda_{2}(s) L}-e^{\lambda_{1}(s) L}}
\end{aligned}
$$

\section{Link with the Characteristics Form}

We observe that in the case where $\gamma=\delta=0$, corresponding to a frictionless horizontal channel, we have $\frac{s}{\lambda_{2}(s)}=\beta$ and $\frac{s}{\lambda_{1}(s)}=-\alpha$. Therefore, we have $\mathcal{X}(s)=\mathbf{X}$, where $\mathbf{X}$ is the matrix used to convert the equations into the characteristics form.

The change of variable $\hat{\zeta}(x, s)=\mathcal{X}(s) \hat{\xi}(x, s)$ can therefore be considered as an extension of the change of variable $\chi(x, t)=\mathbf{X} \xi(x, t)$ leading to the characteristics form. Indeed, the two changes of variable enable to diagonalize the system of equations. However, the change of variable $\hat{\zeta}(x, s)=\mathcal{X}(s) \hat{\xi}(x, s)$ is a dynamic one, since $\mathcal{X}(s)$ depends on the Laplace variable $s$.

The solutions in terms of the generalized characteristic variables is given by:

$$
\begin{aligned}
& \hat{\zeta}_{1}(x, s)=e^{\lambda_{1}(s) x} \hat{\zeta}_{1}(0, s) \\
& \hat{\zeta}_{2}(x, s)=e^{-\lambda_{2}(s)(L-x)} \hat{\zeta}_{2}(L, s)
\end{aligned}
$$

We see here that the diagonal form can also be interpreted as traveling waves: one traveling downstream at "speed" $-\frac{s}{\lambda_{1}(s)}$, the other one traveling upstream at "speed" $\frac{s}{\lambda_{2}(s)}$.

Moreover, it is possible to derive explicit expressions in the time domain for the characteristics variables.

1) Explicit Expressions for the Characteristics: The change of variable enables to diagonalize the system. Finally, the solution in terms of the new characteristics variables can be obtained directly in the frequency domain using Eqs. (20). These expressions can also be translated into the time domain. We introduce the filters $\hat{F}(s)$ and $\hat{H}(l, s)$ defined by:

$$
\begin{aligned}
\hat{F}(s) & =s+b-\sqrt{(s+b)^{2}-a^{2}} \\
\hat{H}(l, s) & =e^{l \hat{F}(s)}-1
\end{aligned}
$$


Using inverse Laplace transform tables [1, pp. 1025-1027], one can check that:

$$
\begin{aligned}
F(t) & =\frac{a e^{-b t}}{t} I_{1}(a t) \\
H(l, t) & =\frac{a l e^{-b t}}{\sqrt{t^{2}-l^{2}}} I_{1}\left(a \sqrt{t^{2}-l^{2}}\right)
\end{aligned}
$$

with $I_{1}(t)$ the modified Bessel function of order 1.

Using Eq. (21), we have:

$$
\begin{aligned}
& \lambda_{1}(s)=-r_{1}-\frac{s}{\alpha}+\frac{(\alpha+\beta)}{2 \alpha \beta} \hat{F}(s) \\
& \lambda_{2}(s)=r_{2}+\frac{s}{\beta}-\frac{(\alpha+\beta)}{2 \alpha \beta} \hat{F}(s)
\end{aligned}
$$

with with $r_{1}=\frac{\alpha \delta-\gamma}{\alpha(\alpha+\beta)}$ and $r_{2}=\frac{\beta \delta+\gamma}{\beta(\alpha+\beta)}$.

Therefore, we have:

$$
\begin{aligned}
e^{\lambda_{1}(s) x} & =e^{-r_{1} x} e^{-\frac{x}{\alpha} s}\left[1+\hat{H}\left(\frac{(\alpha+\beta)}{2 \alpha \beta} x, s\right)\right] \\
e^{-\lambda_{2}(s)(L-x)} & =e^{-r_{2}(L-x)} e^{-\frac{L-x}{\beta} s}\left[1+\hat{H}\left(\frac{(\alpha+\beta)}{2 \alpha \beta}(L-x), s\right)\right]
\end{aligned}
$$

Applying inverse Laplace transform to Eqs. (20) enables us to express the generalized characteristics as a function of their values at the boundary:

$$
\begin{aligned}
& \zeta_{1}(x, t)=e^{-r_{1} x}\left[\zeta_{1}\left(0, t-\frac{x}{\alpha}\right)\right. \\
& \left.\quad+\int_{0}^{t} H\left(\frac{(\alpha+\beta)}{2 \alpha \beta} x, t-\tau\right) \zeta_{1}\left(0, \tau-\frac{x}{\alpha}\right) d \tau\right] \\
& \zeta_{2}(x, t)=e^{-r_{2}(L-x)}\left[\zeta_{2}\left(L, t-\frac{(L-x)}{\beta}\right)\right. \\
& \left.\quad+\int_{0}^{t} H\left(\frac{(\alpha+\beta)}{2 \alpha \beta}(L-x), t-\tau\right) \zeta_{2}\left(L, \tau-\frac{(L-x)}{\beta}\right) d \tau\right]
\end{aligned}
$$

The expressions (26) generalize the ones already obtained in the horizontal frictionless case. The variable $\zeta_{1}(x, t)$ is obtained as the sum of its delayed value at $x=0$ plus its delayed convolution with an infinite dimensional kernel $H(l, t)$, multiplied by a damping coefficient $e^{-r_{1} x}$.

2) Explicit Expressions in the Time Domain: We now use the expressions of the complex eigenvalues $\lambda_{1}(s)$ and $\lambda_{2}(s)$ to obtain the explicit expression of the generalized characteristics for the uniform flow case. The generalized characteristics variables are given by:

$$
\begin{aligned}
& \hat{\zeta}_{1}(x, s)=\hat{q}(x, s)+\frac{T_{0} s}{\lambda_{2}(s)} \hat{y}(x, s) \\
& \hat{\zeta}_{2}(x, s)=\hat{q}(x, s)+\frac{T_{0} s}{\lambda_{1}(s)} \hat{y}(x, s)
\end{aligned}
$$

Using Eqs. (25), expanding $s / \lambda_{1}(s)$ and $s / \lambda_{2}(s)$ and rearranging, we get:

$$
\begin{aligned}
& \frac{s}{\lambda_{2}(s)}=\beta-\frac{\beta^{2} r_{2}}{s+\delta}-\frac{(\alpha+\beta)}{2(s+\delta)} \hat{F}(s) \\
& \frac{s}{\lambda_{1}(s)}=-\alpha+\frac{\alpha^{2} r_{1}}{s+\delta}+\frac{(\alpha+\beta)}{2(s+\delta)} \hat{F}(s)
\end{aligned}
$$

We now use the inverse Laplace transform to express the generalized characteristics $\zeta(x, t)$ as follows:

$$
\begin{aligned}
\zeta_{1}(x, t)= & \chi_{1}(x, t)-\beta^{2} r_{2} T_{0} \tilde{y}(x, t) \\
& -\frac{T_{0}(\alpha+\beta)}{2} \int_{0}^{t} F(t-\tau) \tilde{y}(x, \tau) d \tau \\
\zeta_{2}(x, t)= & \chi_{2}(x, t)+\alpha^{2} r_{1} T_{0} \tilde{y}(x, t) \\
& +\frac{T_{0}(\alpha+\beta)}{2} \int_{0}^{t} F(t-\tau) \tilde{y}(x, \tau) d \tau
\end{aligned}
$$

where $\chi_{1}(x, t)=q(x, t)+\beta T_{0} y(x, t)$ and $\chi_{2}(x, t)=$ $q(x, t)-\alpha T_{0} y(x, t)$ are the Riemann invariants for the solution in the horizontal frictionless case (see Eqs. (2)), $\tilde{y}(x, t)$ is defined by:

$$
\tilde{y}(t)=\int_{0}^{t} e^{-\delta(t-\tau)} y(x, \tau) d \tau,
$$

and $F(t)$ is the convolution kernel given by Eq. (23).

We therefore observe that even if the characteristics approach fails to diagonalize the system with a static change of variable, it is still possible to diagonalize the system, with a dynamic change of variable. This dynamic change of variable can be considered as the extension of the change of variable that leads to the Riemann invariants for the horizontal frictionless channel case.

The change of variable includes a first order filter of the water level, and a more complex infinite dimensional filter with convolution kernel $F(t)$.

We have therefore obtained a dynamic change of variable which is the extension of the characteristic form in the horizontal frictionless case. This change of variable includes a first order filter, and an infinite dimensional filter, which are both linked to the nonzero values of parameters $\gamma$ and $\delta$. When $\gamma=\delta=0$, we recover the horizontal frictionless case, and the variables $\zeta(x, t)$ coincide with the characteristics variables $\chi(x, t)$.

3) Inverse Transform: The inverse transform can also be expressed in the time domain as follows. Using the inverse transformation $\hat{\xi}(x, s)=\mathcal{X}(s)^{-1} \hat{\zeta}(x, s)$, we have:

$$
\begin{aligned}
\hat{y}(x, s) & =\frac{\lambda_{1}(s) \lambda_{2}(s)}{T_{0} s\left(\lambda_{1}(s)-\lambda_{2}(s)\right)}\left(\hat{\zeta}_{1}(x, s)-\hat{\zeta}_{2}(x, s)\right) \\
\hat{q}(x, s) & =\frac{1}{\lambda_{1}(s)-\lambda_{2}(s)}\left(\lambda_{1}(s) \hat{\zeta}_{2}(x, s)-\lambda_{2}(s) \hat{\zeta}_{1}(x, s)\right)
\end{aligned}
$$

Standard manipulations lead to

$$
\begin{aligned}
\hat{y}(x, s)= & \frac{1}{T_{0}(\alpha+\beta)} \Delta \hat{\zeta}(x, s) \\
& +\frac{1}{T_{0}(\alpha+\beta)}\left[\frac{\hat{F}(s)+c_{y}}{\sqrt{(s+b)^{2}-a^{2}}}\right] \Delta \hat{\zeta}(x, s) \\
\hat{q}(x, s)= & \frac{1}{\alpha+\beta}\left(\alpha \hat{\zeta}_{1}(x, s)+\beta \hat{\zeta}_{2}(x, s)\right) \\
& +\frac{\alpha-\beta}{2(\alpha+\beta)}\left[\frac{\hat{F}(s)+c_{q}}{\sqrt{(s+b)^{2}-a^{2}}}\right] \Delta \hat{\zeta}(x, s)
\end{aligned}
$$


with

$$
\begin{aligned}
c_{y} & =\frac{\left(\alpha^{2}+\beta^{2}\right) \delta-(\alpha-\beta) \gamma}{(\alpha+\beta)^{2}} \\
c_{q} & =\frac{2 \alpha \beta}{(\alpha+\beta)^{2}}(2 \gamma-(\alpha-\beta) \delta) .
\end{aligned}
$$

and $\Delta \hat{\zeta}(x, s)=\left(\hat{\zeta}_{1}(x, s)-\hat{\zeta}_{2}(x, s)\right)$

Using inverse Laplace transform tables [1], we find:

$$
\mathcal{L}^{-1}\left\{\frac{\hat{F}(s)}{\sqrt{(s+b)^{2}-a^{2}}}\right\}=a e^{-b t} I_{1}(a t)
$$

and

$$
\mathcal{L}^{-1}\left\{\frac{1}{\sqrt{(s+b)^{2}-a^{2}}}\right\}=e^{-b t} I_{0}(a t)
$$

with $I_{0}(t)$ and $I_{1}(t)$ the modified Bessel functions of order 0 and 1 , respectively.

Therefore, the inverse transformation from $\left(\zeta_{1}(x, t), \zeta_{2}(x, t)\right)$ to $(y(x, t), q(x, t))$ can be expressed as:

$$
\begin{aligned}
y(x, t) & =\frac{1}{T_{0}(\alpha+\beta)} \Delta \zeta(x, t) \\
& +\frac{a}{T_{0}(\alpha+\beta)} \int_{0}^{t} e^{-b(t-\tau)} I_{1}(a(t-\tau)) \Delta \zeta(x, \tau) d \tau \\
& +\frac{c_{y}}{T_{0}(\alpha+\beta)} \int_{0}^{t} e^{-b(t-\tau)} I_{0}(a(t-\tau)) \Delta \zeta(x, \tau) d \tau \\
q(x, t) & =\frac{1}{\alpha+\beta}\left[\alpha \zeta_{1}(x, t)+\beta \zeta_{2}(x, t)\right] \\
& +\frac{a(\alpha-\beta)}{2(\alpha+\beta)} \int_{0}^{t} e^{-b(t-\tau)} I_{1}(a(t-\tau)) \Delta \zeta(x, \tau) d \tau \\
& +\frac{c_{q}(\alpha-\beta)}{2(\alpha+\beta)} \int_{0}^{t} e^{-b(t-\tau)} I_{0}(a(t-\tau)) \Delta \zeta(x, \tau) d \tau .
\end{aligned}
$$

with $\Delta \zeta(x, t)=\left[\zeta_{1}(x, t)-\zeta_{2}(x, t)\right]$.

This enables us to compute the physical variables $y(x, t)$ and $q(x, t)$ as functions of the characteristics variables $\zeta_{1}(x, t)$ and $\zeta_{2}(x, t)$.

Together, the formulas (29), (26) and (31) provide an explicit solution to the linearized Saint-Venant equations around a uniform flow regime. Indeed, assuming zero initial conditions (but nonzero initial conditions could also easily be introduced), one may first use (29) to transform the system into the generalized characteristic variables $\left(\zeta_{1}(x, t), \zeta_{2}(x, t)\right)$. One can then compute the solution given the boundary conditions, using Eqs. (26), and finally, go back to the physical variables $y(x, t)$ and $q(x, t)$ using (31).

\section{Motion Planning}

In this section, we design a boundary controller that enables to reach any pre-specified state $\xi\left(x, T_{r}\right)$ at a given time instant $T_{r}$. This is closely related to the exact controllability problem. In the horizontal frictionless case, one may show that this is possible if $T_{r}$ is greater or equal to $\tau_{2}$. We extend here this exact controllability result to the case of uniform open channel flow with nonzero slope and friction.

\section{A. Explicit Expressions for the Boundary Controls}

Using Eqs. (26), we can express the desired final state $\zeta_{T_{r}}(x)$ as a function of the boundary controls as follows:

$$
\begin{aligned}
& \zeta_{1 T_{r}}(x)=e^{-r_{1} x}\left[\zeta_{1}\left(0, T_{r}-\frac{x}{\alpha}\right)\right. \\
& \left.+\int_{0}^{T_{r}} H\left(\theta x, T_{r}-\tau\right) \zeta_{1}\left(0, \tau-\frac{x}{\alpha}\right) d \tau\right] \\
& \zeta_{2 T_{r}}(x)=e^{-r_{2}(L-x)}\left[\zeta_{2}\left(L, T_{r}-\frac{(L-x)}{\beta}\right)\right. \\
& \left.\quad+\int_{0}^{T_{r}} H\left(\theta(L-x), T_{r}-\tau\right) \zeta_{2}\left(L, \tau-\frac{(L-x)}{\beta}\right) d \tau\right]
\end{aligned}
$$

with $H(l, t)$ given by (24) and $\theta=\frac{(\alpha+\beta)}{2 \alpha \beta}$. With the changes of variables $t=T_{r}-\frac{x}{\alpha}$ for $\zeta_{1}$ and $t=T_{r}-\frac{L-x}{\beta}$ for $\zeta_{2}$, we obtain the following expressions:

$$
\begin{aligned}
& \zeta_{1 T_{r}}\left(\alpha\left(T_{r}-t\right)\right)=e^{-r_{1} \alpha\left(T_{r}-t\right)}\left[\zeta_{1}(0, t)\right. \\
& \left.+\int_{0}^{T_{r}} H\left(\theta \alpha\left(T_{r}-t\right), T_{r}-\tau\right) \times \zeta_{1}\left(0, \tau-T_{r}+t\right) d \tau\right] \\
& \zeta_{2 T_{r}}\left(L-\beta\left(T_{r}-t\right)\right)=e^{-r_{2} \beta\left(T_{r}-t\right)}\left[\zeta_{2}(L, t)\right. \\
& \left.\quad+\int_{0}^{T_{r}} H\left(\theta \beta\left(T_{r}-t\right), T_{r}-\tau\right) \times \zeta_{2}\left(L, \tau-T_{r}+t\right) d \tau\right]
\end{aligned}
$$

Then, choosing $\zeta_{1}(0, t)$ such that:

$$
\zeta_{1}(0, t)= \begin{cases}0 & \text { if } t<T_{r}-\frac{L}{\alpha} \\ e^{r_{1} \alpha\left(T_{r}-t\right)} \zeta_{1 T_{r}}\left(\alpha\left(T_{r}-t\right)\right) & \\ -\int_{0}^{T_{r}} H\left(\theta \alpha\left(T_{r}-t\right), \tau\right) & \\ \times \zeta_{1}(0, t-\tau) d \tau & \text { if } t \geq T_{r}-\frac{L}{\alpha}\end{cases}
$$

ensures that $\zeta_{1}\left(x, T_{r}\right)=\zeta_{1 T_{r}}(x)$ for all $x \in[0, L]$.

And choosing $\zeta_{2}(L, t)$ such that:

$\zeta_{2}(L, t)= \begin{cases}0 & \text { if } t<T_{r}-\frac{L}{\beta} \\ e^{r_{2} \beta\left(T_{r}-t\right)} \zeta_{2 T_{r}}\left(L-\beta\left(T_{r}-t\right)\right) & \\ -\int_{0}^{T_{r}} H\left(\theta \beta\left(T_{r}-t\right), \tau\right) & \text { if } t \geq T_{r}-\frac{L}{\beta} \\ \times \zeta_{2}(L, t-\tau) d \tau & \end{cases}$

ensures that $\zeta_{2}\left(x, T_{r}\right)=\zeta_{2 T_{r}}(x)$ for all $x \in[0, L]$.

These expressions give a solution to the motion planning problem for an open channel, but in an implicit form.

\section{FeEdBack Control}

We now consider boundary feedback control of the open channel, and look for stability conditions. 


\section{A. Expressions in the Characteristics Variables}

The boundary control inputs $(\hat{q}(0, s), \hat{q}(L, s))$ are related to the boundary measurements as follows:

$$
\left(\begin{array}{c}
\hat{q}(0, s) \\
\hat{q}(L, s)
\end{array}\right)=K(s)\left(\begin{array}{c}
\hat{y}(0, s) \\
\hat{y}(L, s)
\end{array}\right)
$$

We now express this relationship in the characteristics variables. Using Eqs. (27), the control inputs in the characteristics are related to the boundary discharges and measurements as follows:

$$
\left(\begin{array}{c}
\hat{\chi}_{1}(0, s) \\
\hat{\chi}_{2}(L, s)
\end{array}\right)=\left(\begin{array}{c}
\hat{q}(0, s) \\
\hat{q}(L, s)
\end{array}\right)+\left(\begin{array}{cc}
\frac{T_{0} s}{\lambda_{2}} & 0 \\
0 & \frac{T_{0} s}{\lambda_{1}}
\end{array}\right)\left(\begin{array}{c}
\hat{y}(0, s) \\
\hat{y}(L, s)
\end{array}\right)
$$

and we also have:

$$
\left(\begin{array}{c}
\hat{\chi}_{1}(L, s) \\
\hat{\chi}_{2}(0, s)
\end{array}\right)=\left(\begin{array}{cc}
0 & 1 \\
1 & 0
\end{array}\right)\left(\begin{array}{c}
\hat{q}(0, s) \\
\hat{q}(L, s)
\end{array}\right)+\left(\begin{array}{cc}
0 & \frac{T_{0} s}{\lambda_{2}} \\
\frac{T_{0} s}{\lambda_{1}} & 0
\end{array}\right)\left(\begin{array}{c}
\hat{y}(0, s) \\
\hat{y}(L, s)
\end{array}\right)
$$

Then, combining with the controller $K(s)$, we get:

$$
\left(\begin{array}{c}
\hat{\chi}_{1}(0, s) \\
\hat{\chi}_{2}(L, s)
\end{array}\right)=G_{K}(s)\left(\begin{array}{c}
\hat{\chi}_{1}(L, s) \\
\hat{\chi}_{2}(0, s)
\end{array}\right)
$$

with $G_{K}(s)$ defined by:

$$
\begin{aligned}
G_{K}(s)= & {\left[K(s)+\left(\begin{array}{cc}
\frac{T_{0} s}{\lambda_{2}} & 0 \\
0 & \frac{T_{0} s}{\lambda_{1}}
\end{array}\right)\right] } \\
& \times\left[\left(\begin{array}{ll}
0 & 1 \\
1 & 0
\end{array}\right) K(s)+\left(\begin{array}{cc}
0 & \frac{T_{0} s}{\lambda_{2}} \\
\frac{T_{0} s}{\lambda_{1}} & 0
\end{array}\right)\right]^{-1}
\end{aligned}
$$

In the case of a diagonal controller, $K=$ $\left(\begin{array}{cc}k_{0}(s) & 0 \\ 0 & k_{L}(s)\end{array}\right)$, we have:

$$
G_{K}(s)=\left(\begin{array}{cc}
0 & \frac{k_{0}(s)+T_{0} s / \lambda_{2}(s)}{k_{0}(s)+T_{0} s / \lambda_{1}(s)} \\
\frac{k_{L}(s)+T_{0} s / \lambda_{1}(s)}{k_{L}(s)+T_{0} s / \lambda_{2}(s)} & 0
\end{array}\right)
$$

\section{B. Stability conditions}

We now use the expressions derived above to study the stability of the system with boundary controls. Let us first recall the input-output relationship in the characteristics variables:

$$
\left(\begin{array}{c}
\hat{\zeta}_{1}(L, s) \\
\hat{\zeta}_{2}(0, s)
\end{array}\right)=G_{P}(s)\left(\begin{array}{c}
\hat{\zeta}_{1}(0, s) \\
\hat{\zeta}_{2}(L, s)
\end{array}\right)+\left(\begin{array}{c}
\bar{\zeta}_{10}(L, s) \\
\bar{\zeta}_{20}(0, s)
\end{array}\right)
$$

with $G_{P}(s)=\left(\begin{array}{cc}e^{\lambda_{1}(s) L} & 0 \\ 0 & e^{\lambda_{2}(s) L}\end{array}\right)$.

Then, we get the following closed-loop relationship:

$$
\left(I-G_{K}(s) G_{P}(s)\right)\left(\begin{array}{c}
\hat{\zeta}_{1}(L, s) \\
\hat{\zeta}_{2}(0, s)
\end{array}\right)=G_{K}(s) G_{0}(s)\left(\begin{array}{c}
\bar{\zeta}_{01}(s, L) \\
\bar{\zeta}_{02}(s, L)
\end{array}\right)
$$

Closed-loop stability can then be studied using the Nyquist theorem, which provides a necessary and sufficient stability condition [3]. However, this condition is not analytic, and is difficult to test. This is why we choose here to propose a simple sufficient condition of stability, based on the spectral radius [3]:
Proposition 1: The closed-loop system is stable if the transfer matrices $G_{P}(s)$ and $G_{K}(s)$ verify the following inequality

$$
\rho_{\max }\left(\left|G_{P}(j \omega) G_{K}(j \omega)\right|\right)<1, \forall \omega \in[0, \infty)
$$

where $\rho_{\max }(A)$ denotes the spectral radius of $A$, i.e. the largest eigenvalue of of $A \in \mathbb{C}^{n \times n}$.

This condition extends the results obtained using Riemann invariants to the case of open channel with slope and friction.

\section{CONCLUSION}

We have derived in this article explicit formulations for solutions of open channel flow including slope and friction effects. These formulas have been obtained by first using the Laplace transform to express the system in the frequency domain, where the system can be diagonalized. This diagonalization extends the classical one leading to Riemann invariants in the horizontal frictionless case. The inverse Laplace transform is then used to go back to the time domain. As a result, we have been able to derive explicit expressions solving the motion planning problem for an open-channel with nonzero slope and friction. Using this approach, we have also extended existing results on the stability of the closed-loop system.

\section{ACKNOWLEDGMENTS}

This paper was written when Xavier Litrico was a Visiting Scholar at the Department of Civil and Environmental Engineering of UC Berkeley, with Professor Alex Bayen. Financial support of Cemagref is gratefully acknowledged.

\section{REFERENCES}

[1] M. Abramowitz and I. A. Stegun. Handbook of mathematical functions, volume 55 of Applied Mathematics Series. National Bureau of Standards, 10th edition, 1972.

[2] J. de Halleux, C. Prieur, J.-M. Coron, B. d'Andréa Novel, and G. Bastin. Boundary feedback control in networks of open-channels. Automatica, 39:1365-1376, 2003.

[3] C. A. Desoer and M. Vidyasagar. Feedback systems: input ouput properties. Academic Press, New York, 1975.

[4] X. Litrico and V. Fromion. Boundary control of hyperbolic conservation laws using a frequency domain approach. In Proc. 45th IEEE Conf. on Decision and Control, San Diego, 2006.

[5] X. Litrico and V. Fromion. Boundary control of linearized Saint-Venant equations oscillating modes. Automatica, 42(6):967-972, 2006.

[6] X. Litrico, V. Fromion, and G. Scorletti. Robust feedforward boundary control of hyperbolic conservation laws. Networks and Heterogeneous Media, 2(4):715-729, 2007.

[7] N. Petit, Y. Creff, and P. Rouchon. Dynamics and solutions to some control problems for water-tank systems. IEEE Trans. Automatic Control, 47(4):594-609, 2002.

[8] C. Prieur, J. Winkin, and G. Bastin. Boundary control of nonhomogeneous systems of two conservation laws. In Proc. 44th IEEE Conf. on Decision and Control, pages 1899-1904, Sevilla, 2005. 